\title{
A digital watermark algorithm based on DCT and improved Watson perceptual model
}

\author{
Shaoyu Chen \\ Department of computer science and technology \\ East China Normal University \\ Shanghai China \\ e-mail:chenshaoyu86@126.com
}

\author{
Xinwei Wang \\ Department of computer science and technology \\ East China Normal University \\ Shanghai China \\ e-mail:wangxw@cs.ecnu.edu.cn
}

\begin{abstract}
An adaptive image watermarking algorithm is proposed based on block Discrete Cosine Transformation(DCT) and the improved perceptual model of Human Visual system(HVS).At first, the digital watermark is transformed by Arnold, then it is effective to make full use of the improved perceptual model in DCT domain and the feature of the subblock luminance to control the strength of the watermark embedded.However, the method can perfect the fidelity of the host image and the robustness of digital watermark. Simulation results demonstrate that the algorithm of watermarking system make a great balance between transparency and robustness, which has a certain practical value.
\end{abstract}

Keywords-digital watermark; DCT; HVS; the improved perceptual model; JND

\section{INTRODUCTION}

With the rapid growth of computer network technology and multimedia processing technology, it is an urgent problem to protect for multimedia information. Digital watermarking is an important method for copyright protection, so how to implement an effective network of copyright has been a great hot spot. Besides, it has a great progress at present.

From the view point of watermark embedding position, digital image watermarking algorithm is usually achieved in time domain or transform domain. At first, the research of digital watermarking is based on time domain. However, we take into account for visual invisibility, and the watermark is often embedded in the least important pixels. Though it is a simple algorithm, it has the poor ability of resisting geometric distortion or noise and the capacity of watermarks to be embedded is quite small. While in transform domain, we firstly transform the image into frequency domain, and then change the coefficients of the frequency domain. Finally, inverse transformation is applied. The most common kinds of transform are DFT, DCT ${ }^{1,11]}$, DWT and so on ${ }^{[6]}$.

This paper focuses on the algorithm of DCT transformation on the basis of fully considering HVS characteristics, and the algorithm based on blocked DCT transformation is quite common. Because the payload of the computation is quite small, it is compatible with the standards of international popular data compression (i.e. JPEG,MPEG,H261/263.etc).Besides, the algorithm is easy to achieve in the compressed domain. Owning to its high robustness, the research of digital watermarking based on the binary value in DCT domain has already been deepened.

\section{DCT TRANSFORM AND THE ANALYSIS OF COEFFICIENTS}

Discrete Cosine Transform (DCT) ${ }^{[3,5]}$ is applied widely in digital image processing area, and is a kind of orthogonal transformation. It comes from the cosine part of the DFT transformation, so it is a real transformation. Therefore, it is good at energy compression and decorrelation and is compatible with video compression standard. In particular, digital watermarking algorithm on the basis of the model of the image's JPEG compression standard can improve the ability of resisting JPEG compression. Consequently, DCT has attracted much attention in the digital watermarking technology.

Two-dimensional (2D) DCT transform is adopted by most image processing. Assuming image $f(x, y)$ is $N \times N$ matrix, its DCT transformation is defined as:

$$
F(u, v)=\alpha_{u} \alpha \sum_{i=0}^{N-1} \sum_{j=0}^{N-1} f(i, j) \cos \left[\frac{(2 i+1) u \pi}{2 N}\right] \cos \left[\frac{(2 j+1) v \pi}{2 N}\right]
$$

Correspondingly, DCT inverse transformation is defined as:

$$
f(i, j)=\sum_{u=0}^{N-1} \sum_{v=0}^{N-1} \alpha_{u} \alpha_{v} F(u, v) \cos \left[\frac{(2 i+1) u \pi}{2 N}\right] \cos \left[\frac{(2 j+1) v \pi}{2 N}\right]
$$

Where $\alpha_{u}, \alpha_{v_{\text {is defined as: }}}$

$$
\alpha_{u}, \alpha_{v}=\sqrt{\frac{1}{N}}, u=0, v=0 \quad \alpha_{u}, \alpha_{v}=\sqrt{\frac{2}{N}}, u=, v=1,2, \ldots, N-1
$$

As mentioned above, while $u=0$ and $v=0$ in formula (1), the coefficient of DCT is known as DC coefficient, and other coefficient is AC coefficients. Two-dimensional (2D) DCT transform can not only make the natural image of the main information concentrate on a small amount of low frequency coefficients, but also cause the minimum of the image blocking effect. In the light of most of image processing, we retain important visual components of the image (i.e. images of the main content), while we mainly change other components of the image (i.e. some details of the image); in corresponding transform domain, the important visual components of the image mainly concentrate on the low frequency part of the image spectrum, while some of the details of the image are mainly distributed in the middle and high frequency of the image 
spectrum. Therefore, the general processing on the image has small effect on the low frequency component, but it has great influence on the high fluency on high component.

To improve the robustness of the digital watermark,cox and so on ${ }^{[4]}$ proposed that the watermark was embedded into the most important components of human visual system(that is, the coefficients of the low frequency domain ).It is possible to cause obvious block effect by changing the coefficient of DC to embed the watermark. Generally speaking, it can't embed the watermark into the coefficient of DC.Therefore, this paper proposes that the embedding positions of the watermark are the component of the middle frequency.

\section{ARNOLD TRANSFORM}

In this paper, the chaotic map is used to encrypt watermark signal, and it become an important mean for digital image transmission and storage. Arnold cat map is employed to shuffle the embedding positions of pixels in host image, which ensures the security of our scheme.

Scrambling transformation has two prominent characteristics: 1) Scrambling transformations are generally periodic. When the chaotic map iterates a certain number of times, it can return to the original map.2) After scrambling transformation, the size of the map don't change. Arnold scrambling (cat mapping) is a transformation that V.J.Arnold ${ }^{[10]}$ presented in ergodic theory research, which is a kind of traditional chaotic system. It is defined as follows:

$$
\left(\begin{array}{l}
x^{\prime} \\
y^{\prime}
\end{array}\right)=\left(\begin{array}{ll}
1 & 1 \\
1 & 2
\end{array}\right)\left(\begin{array}{l}
x \\
y
\end{array}\right)(\bmod N)_{x, y \in\{0,1,2 \ldots, N-1\}}
$$

Where $\mathrm{N}$ denotes the order number (i.e. the size of the

map, usually considering square images), $\left(\begin{array}{l}x \\ y\end{array}\right)$ denotes the position of the pixels before Scrambling transformation, $\left(\begin{array}{l}x^{\prime} \\ y^{\prime}\end{array}\right)$ denotes the position of the pixels after scrambling transformation.

\section{IV.THE IMPROVED WATSON'S PERCEPTUAL MODEL}

According to the difference of each person, human visual system $^{[7]}$ is also different from the sensitive level of the image. Owing to the invisibility of digital watermark, it's requisite to establish the system of the good HVS model ${ }^{[8]}$.At the same time, we must make a large number of experiments. In the procedure of graphical coding, we make full use of the effect of the human masking so as to embed into much more information. Human visual system (HVS) ${ }^{[9]}$ has such characteristics, which are as follows: 1) Sensitivity to the luminance: in a homogeneous background, human eyes are not sensitive to the quite bright or black regions; 2) Contrast masking: in the regions with strong contrast, such as the edge regions, human eyes have a high visual threshold; 3) Textures masking: in the regions with complicated textures, human eyes have a higher visual threshold than that in the flat regions. The sensitivity to the luminance can be measured by the gray values. The regions in host images with strong or weak gray values can be embedded with strong watermarking. The density of edge pixels can measure the contrast and a texture, i.e.

the higher density demonstrates the higher contrast and more complicated textures.

$J_{N D}{ }^{[12]}$ (Just Noticeable Difference) is the very important concept of perceptual model, which is the threshold of the changed signal. While embedding into the watermark, we must meet the demand that the embedded strength of each pixel can't exceed one unit of JND.In the year of 1993, Watson A $\mathrm{B}^{[2]}$ proposed the visual model based on DCT and the concept of JND.The perceptual model consists of sensitivity, luminance masking, contrast masking, and a pooling. As for the coefficients of DCT transform, Human visual system (HVS) has three characteristics: contrast sensitivity (TC), luminance sensitivity (TL), frequency sensitivity (TF):

$$
t_{L}[i, j, k]=t(i, j)\left(C_{0}[0,0, K] / C_{0,0}\right)^{\alpha T}
$$

where $\alpha T$ is a constant, wherein they suggest a value of $0.649, C_{0}[0,0, k]$ is DC coefficient of the kth block in the original image, $C_{0,0}$ is an average of the DC terms for the complete image. And $t[i, j]$ is the sensitivity table that is defined by Watson perceptual model, which reflect human eye sensitivity of different frequency.

$$
s[i, j, k]=\max \left\{t_{L}[i, j, k],\left|C_{0}[i, j, k]\right|^{w[i, j]} t_{L}[i, j, k]^{1-w(i, j)}\right\}
$$

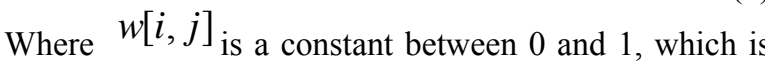
different from the coefficient of the frequency, but a typical empirical value, is 0.7 . But in the improved model

$$
\begin{aligned}
& \left.s[i, j, k]=\min t_{L}[i, j, k], C_{0}[i, j, k]^{w[i, j]} t_{L}[i, j, k]^{1-w[i, j]}\right\} \\
& \text { Where } \alpha T \text { is } 0.849 \text {, and } w[i, j] \text { is } 0.01 .
\end{aligned}
$$

\section{WATERMARK EMBEDDING}

During the procedure of watermark embedding, we apply a kind of technique on the basis of the middle frequencies of DCT.According to the research of HVS, we found that human eyes are more sensitive to the low frequency part of the nosies.To improve the invisibility of digital watermark, we can embed the watermark into the high frequency part of the host image, but it is easy to miss the information of digital watermark by quantification, low pass filtering and so on.Thus, the means of embedding watermark have a negative effect on the robustness of the watermark.Consequently,it is a tradeoff between robustness and invisibility to choose the middle frequency part of the host image.

\begin{tabular}{|l|l|l|l|l|l|l|l|}
\hline 0 & 1 & 5 & 6 & 14 & 15 & 27 & 28 \\
\hline 2 & 4 & 7 & 13 & 16 & 26 & 29 & 42 \\
\hline 3 & 8 & 12 & 17 & 25 & 30 & 41 & 43 \\
\hline 9 & 11 & 18 & 24 & 31 & 40 & 44 & 53 \\
\hline 10 & 19 & 23 & 32 & 39 & 45 & 52 & 54 \\
\hline 20 & 22 & 33 & 38 & 46 & 51 & 55 & 60 \\
\hline 21 & 34 & 37 & 47 & 50 & 56 & 59 & 61 \\
\hline 35 & 36 & 48 & 49 & 57 & 58 & 62 & 63 \\
\hline
\end{tabular}

$8 \times 8$ zig-zag order 
The positions of the original image are the part of the matrix in which the position of the value is 1 , the matrix is as follows:

midband $=[$

$0,0,0,0,0,0,0,0$

$0,0,0,0,0,0,0,0$

$0,0,0,0,0,0,0,0$

$0,0,0,1,1,0,0,0$

$0,0,0,1,1,0,0,0$

$0,0,0,0,0,0,0,0$

$0,0,0,0,0,0,0,0$

$0,0,0,0,0,0,0,0]$;

The step of embedding algorithm is as follows:

(1)The binary watermark image is transformed into $\mathrm{M}^{\prime}$ by Arnold transformation to lower the autocorrelation coefficients of the image, and retain the key $\mathrm{K}$.

(2)The host image $f(x, y)$ that its size is $\mathrm{N} \times \mathrm{N}$ is divided into $8 \times 8$ blocks block $(\mathrm{x}, \mathrm{y})(\mathrm{x}, \mathrm{y}=1,2, \ldots 8)$.According to the improved Watson's perceptual model, we can gain the JND matrix of each block (that is, ths matrix).

(3)The original image is partitioned into non-overlapping blocks, and block_det(x,y)is getted after block $(\mathrm{x}, \mathrm{y})$ is transformed into DCT domain.

(4)Let us read the scrambling binary image $M^{\prime}$, if $M^{\prime}(i, j)=0$, then $\mathrm{a}=1$, otherwise $\mathrm{a}=-1$. According to the location of the embedded pixels that the value in matrix called midband is 1 , we change the coefficients of the host image, the formula is given by:

block $\operatorname{det}(\mathrm{j}, \mathrm{i})=$ block $\operatorname{det}(\mathrm{j}, \mathrm{i}) *(1+\mathrm{a}(\mathrm{j}, \mathrm{i}) *$ ths $(\mathrm{j}, \mathrm{i})$;

(5)After block $\operatorname{dct}(\mathrm{j}, \overline{\mathrm{i}})$ is modified, watermarking image is achieved when inverse DCT transformation is applied in each block.

(6)Merging the image of each block, we can get the watermark embedded image I W.In addition, we make public the embedded locations of the matrix called midband.

\section{WATERMARK EXTRACTION}

(1)The watermark embedded image I_W and the host image are divided into $8 \times 8$ non-overlapping blocks, respectively.Then, we transform them into DCT domain (i.e. block_det1 and block_det).

(2)According to the positions of the embedded watermarking image, we employ this formula. The formula is given by:

aa=block_det1/block_det-1

If aa $>=0, w^{\prime}(i)=1$, otherwise $w^{\prime}(i)=0$;

(3)After we deal with the watermark embedded image of each block, we obtain the scrambling watermark image $\mathrm{W}^{\prime}$.According to the key $\mathrm{K}$, the watermark signal is achieved after $\mathrm{W}^{\prime}$ is transformed by Arnold.

\section{SIMULATION RESULTS}

The simulations are performed in Matlab.Peak signal to noise ratio (PSNR) ${ }^{[6]}$ is used to measure the invisibility of the embedded watermark and normalized cross correlation
(NC) is used to measure the similarity between the extracted watermark and the original watermark. The formulas are given by:

$$
\text { PSNR }=10 \log 10 \frac{M N \max \left[\max \left(I^{2}\right)\right]}{\sum_{i=1}^{i=M} \sum_{j=1}^{j=N}\left[I(i, j)-I^{\prime}(i, j)\right]^{2}}
$$

Where $\mathrm{I}$ is the host image, $\mathrm{I}$ ' is the image embed with watermark, $\mathrm{M} \times \mathrm{N}$ is the size of $\mathrm{I}$.

$$
N C=\frac{\sum_{i=1} \sum_{j=N}^{j=M} W(i, j) W W^{\prime}(i, j)}{\sum_{i=1}^{i=M} \sum_{j=1}^{j=M} W(i, j)^{2}}
$$

Where $\mathrm{W}$ denotes the original watermark, W' denotes the extracted watermark.

One $256 \times 256$ gray images 'Lena' is used for testing in Figure 1(a), and a size of $32 \times 32$ binary image shown in Figure 1(b) is selected as watermark.

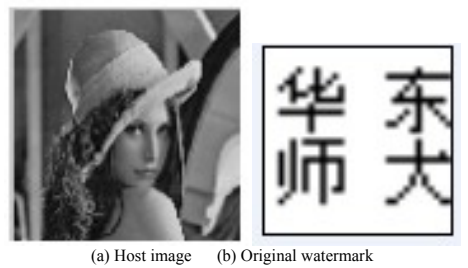

Figure1 Gray carrier image and watermark image

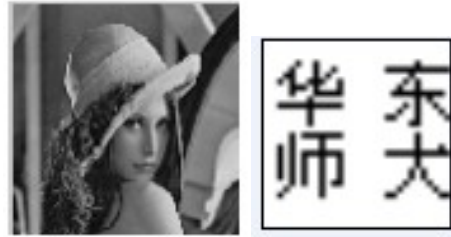

(c)Image embedded (d) Watermark extracted

With watermark under normal environment

Figure 2 Image embedded with watermark and watermark extracted under normal environment.

When the watermark is extracted under normal environment, $\mathrm{NC}=1$ and $\mathrm{PSNR}=64 \mathrm{db}$. To test the robustness of the algorithm, a sequence of attack experiments with different level of strength are applied to the image embedded with watermark. The attack experiments are as follows:

(1)JPEG compression

\begin{tabular}{|l|l|l|}
\hline Quality factor & NC & PSNR \\
\hline $80 \%$ & 0.9997 & 45.3672 \\
\hline $60 \%$ & 0.9994 & 44.7452 \\
\hline $40 \%$ & 0.9993 & 44.6219 \\
\hline $10 \%$ & 0.9989 & 42.8051 \\
\hline
\end{tabular}

(2)Filtering

\begin{tabular}{|l|l|l|}
\hline The kind of filter & NC & PSNR \\
\hline Median filter & 0.9991 & 42.9963 \\
\hline Gaussian & 0.9986 & 41.8547 \\
\hline
\end{tabular}

(3)noise contamination

\begin{tabular}{|c|l|l|}
\hline The kind of noise & NC & PSNR \\
\hline Gaussian noise & 0.9946 & 49.4689 \\
\hline Salt \& Pepper noise & 0.9948 & 44.5986 \\
\hline
\end{tabular}

The watermarks extracted from the watermarking images which are contaminated by Gaussian noise whose average value is 0 and variance is 0.001 , and Salt \& Pepper noise whose strength is 0.01 .If we comply with the rules 
that $\mathrm{NC} \geq 0.750$, we can obtain an effective watermark.

(4)Image cropping

\begin{tabular}{|c|l|l|}
\hline $\begin{array}{c}\text { The } \\
\text { percentage of the image } \\
\text { cropping }\end{array}$ & NC & PSNR \\
\hline $1 / 4$ & 0.9989 & 43.2751 \\
\hline $1 / 2$ & 0.9978 & 42.4931 \\
\hline
\end{tabular}

\section{CONCLUSION}

An improved Watson's perceptual model image watermarking has been introduced in this paper. The paper proposes an adaptive robust watermarking algorithm in DCT domain associating with scrambling technology, HVS and DCT transform. The watermark is embedded into a digital image, by modifying the middle frequency coefficients in DCT domain .In spite of the method's simplicity, the embedded watermark can successfully survive after attacked a series of image processing operations. At the same time, we can get a compromise between transparency, robustness and capacity.

\section{REFERENCES}

[1]SUN ShengHe,LU ZheMing,NIU XiaMu.Digital watermarking technology and its application .Beijing: Science Press, 2004

[2] Andrew B Watson .Visually optimal DCT quantization matrices for individual images [J].Data Compression Conference, 1993. DCC'93.
[3] Andrew B Watson .DCT Quantization Matrices optimalized for Individual Images [J]. Human Vision, Visual Processing, and Digital Display IV, 2002, 19(13):202-216.

[4] HUANG JiWu, Yun Q. SHI,CHENG WeiDong. Image Watermarking in DCT: An Embedding Strategy and Algorithm [J].ACTA ELECTRONICA SINICA, 2000, 28(4):57-60.

[5] I.J Cox, Joe Kilian, FT Leighton, et al.Secure spread spectrum watermarking for multimedia [J].Image Processing, 1997, 1673-1687

[6]NIU XinYue.Information hiding and digital watermarking .Beijing University of Posts and Telecommunications press, 2004.6.

[7]LING Hefei,LU ZhengDing,ZOU FuHao,LI RuiXuan.An Energy Modulated Watermarking Algorithm Based on Watson Perceptual Model [J].Journal of Software.2006.05.

[8]Ahmed A.Abdulfetah, Xingming Sun, Hengfu Yang and Nur Mohammad.Robust adaptive image watermarking using visual models in DWT and DCT domain [J].Information Technology Journal 9(3):460-466, 2010

[9] Qiao Li and Ingemar J. Cox. Using Perceptual Models to Improve Fidelity and Provide Resistance to Volumetric Scaling for Quantization Index Modulation Watermarking [J].Information Forensics and Security 2(2):127-139, 2007

[10]Wang Sheng Fang, Lu Lu Wu, Rong Zhang. A Watermark Preprocessing Algorithm Based on Arnold Transformation and Logistic Chaotic Map [J].Advanced Materials Research (Vols 341 - 342) 720- 724, 2012

[11]I. J. Cox,M. L. Miller,J. A. Bloom. Digital Watermarking. Morgan Kaufmannn,2002.

[12]Cong Jin,Feng Tao,Yu Fu.Image watermarking based HVS characteristic of wavelet transform[J]. 2006 International Conference on Intelligent Information Hiding and Multimedia Signal Processing,Pasadena, California,2006,71-74. 